\title{
ON IRREDUCIBLE SYSTEMS OF ALGEBRAIC DIFFERENTIAL EQUATIONS
}

\author{
BY E. GOURIN
}

Given two irreducible algebraic manifolds, if the first is a proper sub-manifold of the second, the first is of lower dimensionality than the second.* We prove here an analogous result for systems of algebraic differential equations.

Terminology and notation will be as in Ritt's monograph, Differential Equations from the Algebraic Standpoint. $\dagger$ Let $\Sigma_{1}$ be a non-trivial closed irreducible system. Let the unknowns be $u_{1}, \cdots, u_{q}, y_{1}, \cdots, y_{p}$, with the $u_{i}$ arbitrary unknowns. We prove the following theorem.

THEOREM. If the manifold of the non-trivial closed irreducible system $\Sigma_{1}$ is a proper sub-manifold of the manifold of another such system $\Sigma_{2}$, then either $\Sigma_{2}$ has a set of arbitrary unknowns of which $u_{1}, \cdots, u_{q}$ form a proper subset, or $u_{1}, \cdots, u_{q}$ form a complete set of arbitrary unknowns of the system $\Sigma_{2}$ and, for these arbitrary unknowns, resolvents of $\Sigma_{1}$ are of lower order than those of $\Sigma_{2} . \ddagger$

Because $\Sigma_{2}$ holds $\Sigma_{1}$, and $\Sigma_{1}$ is closed, every form of $\Sigma_{2}$ is contained in $\Sigma_{1}$. Then, certainly, $\Sigma_{2}$ cannot contain a form involving the $u_{i}$ alone. Otherwise $\Sigma_{1}$ would contain such a form. Consequently, there exists in $\Sigma_{2}$ a set of arbitrary unknowns of which $u_{1}, \cdots, u_{q}$ form a subset. This subset is either a proper subset or a full set of arbitrary unknowns of $\Sigma_{2}$.

Let us assume, then, that this latter condition is satisfied, that is, $u_{1}, \cdots, u_{q}$ form a complete set of arbitrary unknowns of both systems $\Sigma_{1}$ and $\Sigma_{2}$.

In order to construct a resolvent of $\Sigma_{2}$ we choose two forms $G$ and $Q$, with $G$ not in $\Sigma_{2}$ and free of the $y_{i}$, such that, for any two distinct solutions of $\Sigma_{2}$ with the same $u_{i}$, such that $G$ does not vanish for these solutions, $Q$ yields two distinct functions

* Van der Waerden, Moderne Algebra, vol. 2, p. 63.

$\dagger$ Published by this Society, 1932.

$\ddagger$ We assume that the associated field contains a non-constant function. This involves no loss of generality. 
of $x$. Having selected two such forms, we adjoin to $\Sigma_{2}$ the form $w-Q$, where $w$ is a new unknown, and obtain a system, say $\Lambda_{2}$. It is clear, however, that the above described properties of the forms $G$ and $Q$ with respect to the system $\Sigma_{2}$ will remain undisturbed when tested with respect to the system $\Sigma_{1}$. Hence, the same forms $G$ and $Q$ may be utilized in the construction of the resolvent of $\Sigma_{1}$. Accordingly, we adjoin the same form $w-Q$ to $\Sigma_{1}$, obtaining a system $\Lambda_{1}$.

Let $\Omega_{1}$ and $\Omega_{2}$ be the two systems of forms in $w$, the $u_{i}$, and $y_{i}$, which vanish for all solutions of $\Lambda_{1}$ and $\Lambda_{2}$, respectively. The two systems $\Omega_{1}$ and $\Omega_{2}$ are closed and irreducible. Furthermore, any form of $\Omega_{2}$ is contained in $\Omega_{1}$.

We now list the unknowns in $\Omega_{1}$ in the order $u_{1}, \cdots, u_{q}$; w; $y_{1}, \cdots, y_{p}$, and take a basic set for $\Omega_{1}$

$$
A, A_{1}, \cdots, A_{p},
$$

in which $w, y_{1}, \cdots, y_{p}$ are introduced in succession and in which $A$ is algebraically irreducible. The equation $A=0$ is a resolvent of $\Omega_{1}$. Each $A_{k}$ is a form in $w$, the $u_{i}$, and $y_{k}$ alone, is of order 0 in $y_{k}$, and of the first degree in $y_{k}$.

Similarly we proceed with $\Omega_{2}$ and take a basic set

$$
B, B_{1}, \cdots, B_{p},
$$

with $B$ algebraically irreducible. Then $B=0$ is a resolvent of $\Omega_{2}$ and each $B_{k}$ is of the same structure as the corresponding $A_{k}$.

It is clear that $A$ cannot be of higher order in $w$ than $B$. Let us assume, then, that $A$ and $B$ are of the same order in $w$. Let $I$ be the initial of $A$. Then, for an appropriate non-negative integer $t$, we have the identity

$$
I^{t} B=A C+D,
$$

where $C$ and $D$ are forms in $w$ and the $u_{i}, D$ being of lower rank in $w$ than $A$. Since $D$ vanishes for every solution of $\Omega_{1}, D$ is contained in $\Omega_{1}$. Hence $D \equiv 0$.

It follows, therefore, from (3) that $I^{t} B$ is divisible by $A$. Because $A$ is algebraically irreducible, and $I$ and $A$ are relatively prime, $B$ must be divisible by $A$. Let $B=A \cdot K$. Then $K$ is a function of $x$, the form $B$ being algebraically irreducible. This implies that in (1) we may replace $A$ by $B$, because, in choosing this basic set, we may select for $A$ among the forms of $\Omega_{1}$ in- 
volving $w$ and the $u_{i}$ alone, any algebraically irreducible form which is of the least rank in w. Such a form, however, was found to be a member of $\Omega_{2}$ and hence may be identified with the form $B$.

$B_{1}$ is contained in $\Omega_{1}$. It is reduced with respect to $B$ and, of all such forms in $\Omega_{1}$ in the $u_{i}$, w, and $y_{1}$, it certainly has a lowest rank. Consequently we may replace $A_{1}$, in (1), by $B_{1}$. Continuing, we find that (2) is a basic set for $\Omega_{1}$. Then $\Sigma_{1}$ and $\Sigma_{2}$ are identical. This contradiction proves that $A$ is of lower order in $w$ than $B$ and establishes our theorem.

Columbia University

\section{AXIOM $C$ OF HAUSDORFF AND THE PROPERTY OF BOREL-LEBESGUE*}

\section{BY SELBY ROBINSON $\dagger$}

1. Introduction. This is a study in an abstract space $(P, K)$ of the Hausdorff $\ddagger$ property $C$ which may be expressed in the form the interior of every set is an open set. A point $p$ of the space $P$ is interior to a set $V$, if $p$ is a point of $V$ and is not a $K$-point (point of accumulation, limit point) of any subset of $C(V)$. An open set is one all of whose points are interior points. We say that space $(P, K)$ has property $B$ of Hausdorff if and only if any point $p$ which is interior to each of two sets is interior to their logical product; we shall designate as the open set $B$ property, the weaker property: the product of two open sets is an open set. $\S$ By the Hausdorff property $D$ we shall mean that any two points are respectively interior to sets which are disjoined, while in the open set $D$ property the points are required to be in disjoined open sets. The Borel and Borel-Lebesgue properties take three non-equivalent forms in spaces not having property $C$. These three forms coincide if property $C$ is present as do the two forms of property $B$ and of property $D$. In $\$ 3$ we consider three

* Presented to the Society, October 29, 1932.

$\dagger$ National Research Fellow.

$\ddagger$ F. Hausdorff, Grundzüge der Mengenlehre, first edition, 1914, p. 213.

$\S$ Chittenden chose the open set $B$ property as the one to designate as the Hausdorff $B$ property. See Transactions of this Society, vol. 31 (1929), p. 315. 